\title{
ON THE EFFECTIVENESS OF MANGROVES IN ATTENUATING CYCLONE - INDUCED WAVES
}

\author{
Siddharth Narayan ${ }^{1}$, Tomohiro Suzuki ${ }^{2}$, Marcel J.F. Stive ${ }^{2}$, Henk Jan Verhagen ${ }^{2}$, W.N.J Ursem ${ }^{3}$, \\ Roshanka Ranasinghe
}

\begin{abstract}
A study of the effectiveness of mangroves in attenuating cyclone- induced waves was done using the SWAN 40.81 numerical model. Hydraulic parameters during extreme events and local mangrove vegetation parameters were estimated for the Kanika Sands mangrove island near the upcoming Dhamra Port in Orissa, India. Simplified generic analyses were first conducted to obtain insights into the characteristics and behaviour of the model and the system. These were used to select relevant scenarios for simulations of actual conditions at the case-study site. The mangroves were found to be effective in reducing wave heights at the port behind the island though the effectiveness is limited by its geometry and distance from the port. The presence of vegetation has a marked effect though the effect of a variation in vegetation factor is limited. An optimum cross-shore width range for maximum protection was quantified.
\end{abstract}

Keywords: Mangroves; Vegetation Factor; Cyclone; Wave Attenuation; Kanika Sands

\section{INTRODUCTION}

Tropical coastlines are under great pressure due to a rapid increase in population and infrastructure. Large-scale mismanagement of these coastlines and the inability to cope with events such as cyclones can have devastating long and short term effects especially in developing countries. It is a wellestablished fact that mangroves help protect the hinterland by attenuating waves during extreme events and reduce long term coastal erosion by trapping sediment (UNEP-WCMC 2006). With increasing population pressure, demand for development on tropical coastlines and the world-wide necessity for environmental protection it is urgent and essential to establish the usefulness of mangroves in protecting ports or other coastal developments from the effects of a tropical cyclone.

While the mechanism of wave attenuation in mangroves has been studied earlier (e.g. Kobayashi et al., 1993; Massel et al., 1999; Mendez \& Losada, 2004), the extent of protection they offer is still not clear due to their inherent complexity and high regional variability. There is however a need to quantify the protective effect of mangroves, examine the controlling parameters and thereby facilitate effective management of these crucial and threatened ecosystems (UNEP-WCMC, 2006).

Recently, the wave attenuation model presented by Mendez and Losada (2004) was extended to incorporate a full frequency-direction wave spectrum in the numerical wave model SWAN and additionally included a layer-wise implementation of vegetation characteristics (Suzuki et al. 2010). This paper uses the extended numerical wave attenuation model to investigate, with respect to several parameters, the effectiveness of mangroves in attenuating cyclone-induced waves and performs a quantitative case study for a mangrove island near the upcoming Dhamra Port in Orissa, India.

\section{BACKGROUND}

\section{Cyclones}

Cyclones are cloud clusters characterized by an organized closed circulation of air, with maximum sustained wind speeds exceeding $121 \mathrm{~km} / \mathrm{hr}$ that develop in the tropical regions of the Indian Ocean (from Fritz \& Blount 2007). Due to the large scale destruction they cause several vulnerability studies of coastal regions in India have been conducted with regard to cyclones. One such study indicates that for the months of October and November Orissa has the highest probability (56\%) among the states on the east coast of India that at least one cyclone makes landfall every year (Mascarenhas A., 2004). Chittibabu et al., 2004 brought together two databases for cyclones in Orissa - one from the India Meteorological Department (IMD) with data from 1877 to 2000 and the other from various records including the British East India Company, the IMD and state government records with data from 1804 to 2000 . 16 cyclone events were modeled using a storm surge model developed by Dube et al. (2000) and verified with available data. The study by Dube et al. (2000) showed that a cyclone similar to the

\footnotetext{
${ }^{1}$ School of Civil Engineering and the Environment, University of Southampton, Southampton, SO17 1BJ, United Kingdom

${ }^{2}$ Faculty of Civil Engineering and Geosciences, Delft University of Technology, Stevinweg 1, 2628 CN DELFT, The Netherlands

${ }^{3}$ Botanical Garden, Delft University of Technology, Julianalaan 67, 2628 BC DELFT, The Netherlands

${ }^{4}$ Department of Water Engineering, UNESCO-IHE, PO Box 3015, 2601 DA Delft, The Netherlands
} 
super-cyclone in Paradip, Orissa in 1999, which claimed around 10,000 lives, had a return period of 47 years. $90 \%$ of the damage caused by a cyclone is attributed to either the accompanying storm surge, waves or precipitation (Chittibabu et al. 2004).

\section{Mangroves}

Mangroves or mangal refer to a coastal inter-tidal ecosystem of halophytic wooded plants that generally occur between mean sea level and the highest spring tidal level. A socio-economic study conducted in Orissa (Das S., 2007) investigating the effect of the Paradip super-cyclone of July 1999 established that coastal villages situated behind mangroves escaped with much less damage compared to villages that did not enjoy their protection. Analytical and numerical wave attenuation models have been proposed that calculate the energy loss in a wave propagating through a vegetation field due to interaction between the vegetation and the wave. The linear wave theory is assumed to be valid throughout the region and inertial forces are generally neglected.

\section{The Model}

Mendez and Losada (2004) proposed a wave dissipation model based on the Dalrymple formulation (Dalrymple et al. 1984), assuming the mangrove vegetation as being similar to a three layer rigid cylindrical structure. The model depends on a parameter similar to drag coefficient parameterized as a function of the Keulegan - Carpenter number for a given plant based on laboratory experiments for different plant types (Mendez \& Losada, 2004). Based on this model a routine was developed and later modified in the numerical model SWAN in the Delft University of Technology (Burger 2005, Suzuki et al. 2010). The SWAN model currently enables the calculation of energy dissipation of a wave propagating through a vegetation field consisting of layered rigid cylinders and is schematized vertically and horizontally to allow for variations in vertical layering as well as spatial density.

\section{The Case-Study Site}

The case - study site is a mangrove inhabited island called the Kanika Sands off the Orissa coast, near the Dhamra river mouth. The newly formed island seen in Figure 1 is oval in shape, roughly $4 \mathrm{~km}$ along the N-S axis and $1.5 \mathrm{~km}$ along the E-W axis at a maximum. The island is around $3.5 \mathrm{~km}$ directly eastward of the upcoming Dhamra Port. The morphology in the region is highly unstable though showing a tendency for progradation. The island has been in existence for more than 30 years and is currently heavily inhabited throughout by mangroves of species such as Avicennia marina, Avicennia alba, Sonneratia alba, Rhizophora mucronata and Phoenix paludosa that were found to attain a height of $10 \mathrm{~m}$ or more (Johnston \& Santillo, 2007).

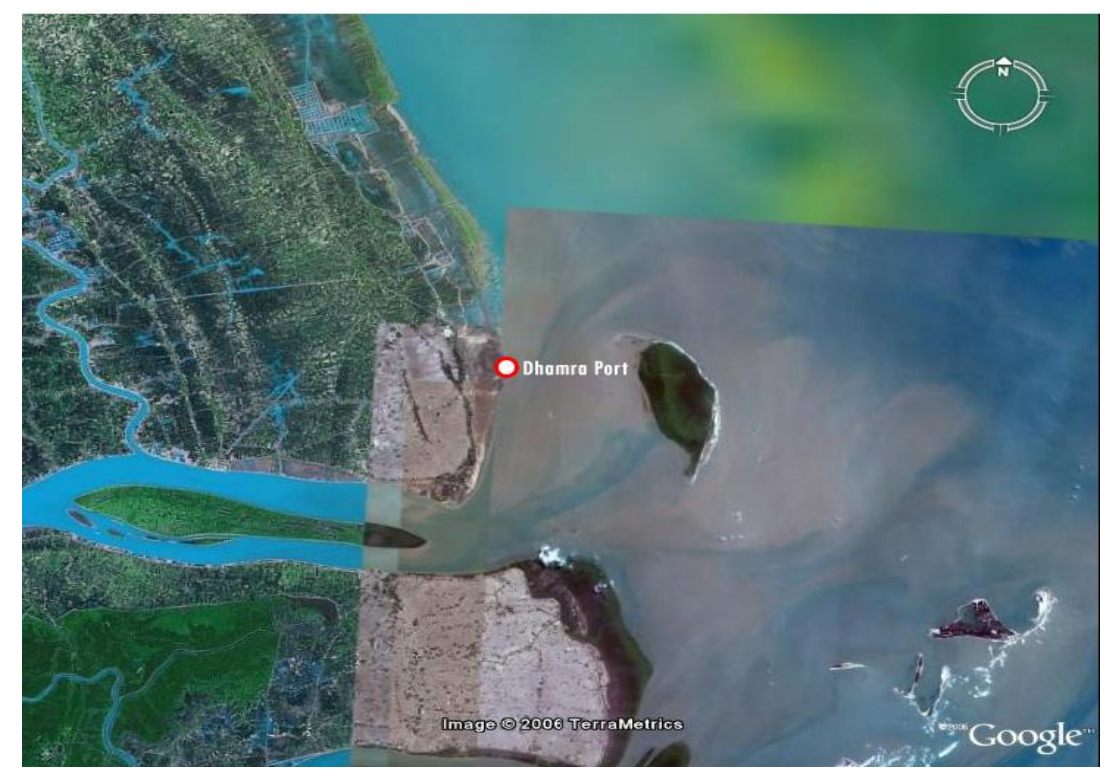

Figure 1: Google Earth Image of Case Study Site (c. 2006) 


\section{MODEL BOUNDARY CONDITIONS}

Nearshore wave heights and periods were estimated using statistics of 13 cyclone events selected from Chittibabu et al. (2004). The estimation involved three stages, namely, calculation of the cyclone parameters, offshore wave parameters and nearshore storm surge levels. The cyclones were assumed to have a constant radius of maximum wind-speed of $45 \mathrm{~km}$, peripheral pressure values of $1012 \mathrm{mb}$ and a velocity of forward movement of $6 \mathrm{~m} / \mathrm{s}$. Different internationally accepted empirical relations between cyclone parameters and wave parameters were examined for the first two stages. The final wave parameters were calculated as the average of the methods that showed acceptable trends and values with the control values obtained from formulae that were derived based on local cyclone events (refer Narayan 2009). Storm surge levels were estimated by extrapolating available data (Jayanti, 1986 in Murthy et al., 2007) for the desired return periods. The derived relationships between offshore wave parameters, surges and return period were validated against measurements in the region.

The 1-D offshore bathymetry for the calculation of wave transformation from deep water to shallow water was estimated using low-scale hydrographic maps. The depth contours were assumed to run more or less parallel to the coast. Offshore wave parameters and water levels were used with the digitized 1-D bathymetry grid in SWAN 1-D for different angles of wave approach to calculate near-shore wave conditions at the boundary of a smaller 2-D grid to be for the generic and case studies. A single Extreme Instantaneous Water Level (EIWL) was used throughout the grid for each return period, equal to the sum of the corresponding storm surge, the local high tide of $3.3 \mathrm{~m}$ (Selvam, 2003) and an estimated value for sea level rise after 80 years. Wave height transformations were calculated for return periods of 5, 10, 25, 50 and 100 years for wave normal angles of $22.5^{\circ}, 45^{\circ}$ and $90^{\circ}$ to the coast. The eastern (seaward) boundary of the grid was assigned a wave height equal to the average wave height across all the angles for the desired return period with the northern and southern boundaries kept open. The calculated nearshore significant wave height, storm surge levels and time periods for the various return periods at the $-11 \mathrm{~m}$ depth contour are shown in Figure 2.

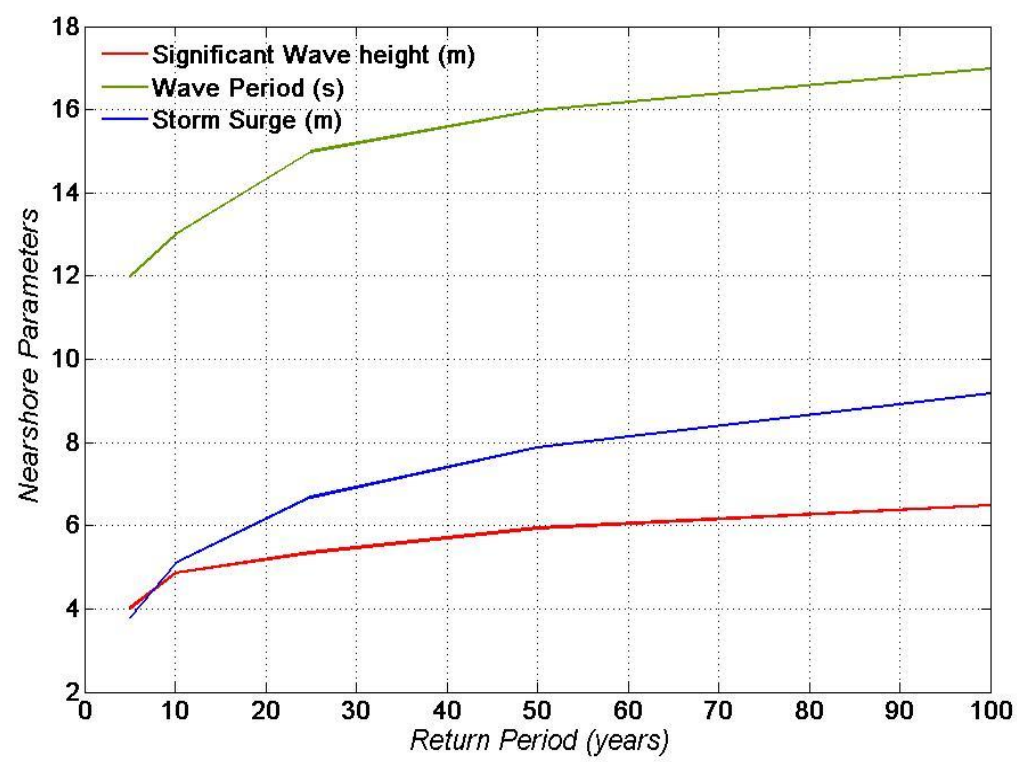

Figure 2: Nearshore significant wave heights (Hs), Storm surge levels (SS) and peak periods (Tp) at the -11 m depth contour

Model vegetation parameters were decided based on the prevalent vegetation in the region, the local bathymetry and the established zonation pattern of mangroves. Based on these factors the vegetation was generalized as belonging to the species $-R$. mucronata. The stem heights were assumed to be 6 to $7 \mathrm{~m}$ based on the assumption that the canopy would remain above the high tide line at all times (Mazda et al. 2007). Details of the island bathymetry, EIWL and vegetation are given in Figure 3 below. The assumed value ranges for the relevant characteristics of the $R$. mucronata species are listed in Table 1. 


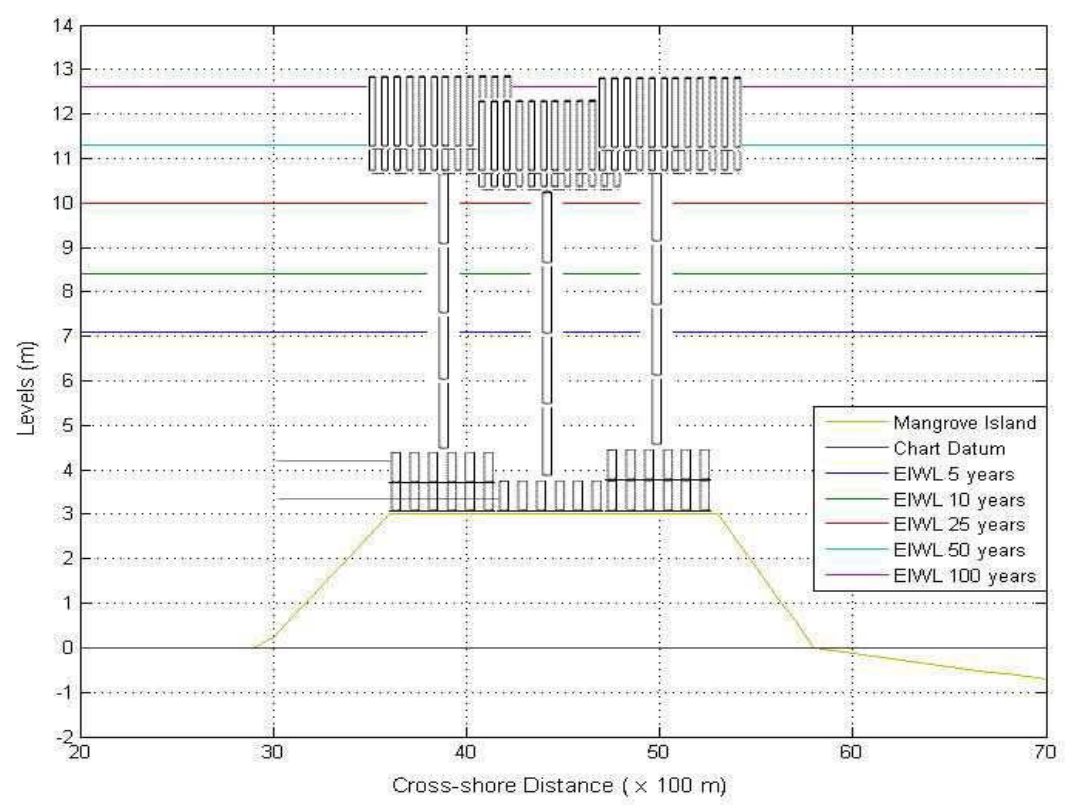

Figure 3: Details of the modelled island cross-section, placement and schematised mangrove vegetation (not to scale) and assumed Extreme Instantaneous Water Levels (EIWLs) for different return periods

\begin{tabular}{|l|l|l|}
\hline \multicolumn{3}{|c|}{ Table 1: Species - R. mucronata (Narayan, 2009) } \\
\hline Parameter & Value Range & Control Value \\
\hline 1. Stem Diameter (DBH) & $0.15-0.4 \mathrm{~m}$ & $0.25 \mathrm{~m}$ \\
2. Root Diameter & $0.05-0.1 \mathrm{~m}$ & $0.075 \mathrm{~m}$ \\
3. Canopy Diameter & $0.02-1 \mathrm{~m}$ & $0.5 \mathrm{~m}$ \\
4. Stem Density & $0.5-1.7$ & 0.7 \\
5. Root Density & $1-130 \mathrm{~m}^{-2}$ & $60 \mathrm{~m}^{-2}$ \\
6. Canopy Density & $1-100 \mathrm{~m}^{-2}$ & $100 \mathrm{~m}^{-2}$ \\
7. Stem Height & $5-8 \mathrm{~m}$ & $6 \mathrm{~m}^{+}$ \\
8. Root Height & $0-1 \mathrm{~m}$ & $0.8 \mathrm{~m}$ \\
9. Canopy Height & $0.2-3 \mathrm{~m}$ & $2 \mathrm{~m}$ \\
\hline
\end{tabular}

\section{GENERIC MODELING STUDIES}

A generic analysis was conducted to obtain insights into the characteristics and behaviour of the model and the system. For this a flat bathymetry was used with the simplified vegetation parameters and calculated wave and water level conditions. The eastern (seaward) boundary of the generic model grid was set at the $-3 \mathrm{~m}$ depth contour. Wave parameters at this boundary were calculated by transformation of values at the $-11 \mathrm{~m}$ depth contour using a 2-D grid with digitized depth contours and a $100 \mathrm{~m}$ by $100 \mathrm{~m}$ resolution. The grid bathymetry was assumed to be flat throughout with a constant height of $3 \mathrm{~m}$ above Chart Datum based on the local high tide level, to enable a more effective study of the influence of the vegetation. The mangrove patch was introduced in the centre of the island as a square of side 4 $\mathrm{km}$. A gap of $2 \mathrm{~km}$ was provided between the vegetation boundary and the grid's seaward boundary to allow the model to adjust to local conditions before encountering vegetation. The input and computational grids were both given a resolution of $100 \mathrm{~m}$ by $100 \mathrm{~m}$.

A total of eighteen scenarios, listed in Table 2, were modeled combining three vegetation parameter, two vegetation height and three hydraulic parameter scenarios. The SWAN model uses a variable called the Vegetation Factor similar to a drag factor to represent the vegetation effect. The vegetation factor here is defined as the product of diameter, density and drag coefficient of each vegetation layer. Based on this the vegetation parameter values in the generic analysis were varied 
corresponding to 'Low' 'Medium' and 'High' vegetation factors for each of the three layers. The 'Low' values were taken from Table 1 and the 'Medium' and 'High' values were calculated as constant multiples of the 'Low' values in order to provide a uniform value range (Narayan, 2009). Two vegetation heights were used, corresponding to emergent and submergent vegetation. Hydraulic parameters - wave height, water level and time period, corresponding to return periods of 100, 25 and 5 years were used. The angle of wave attack was kept constant at $90^{\circ}$ for all the runs.

\begin{tabular}{|c|c|c|}
\hline \multicolumn{3}{|c|}{ Table 2: Modeled scenarios for generic sensitivity analyses } \\
\hline $\begin{array}{l}\text { Vegetation Factor } \\
(3 \text { cases })\end{array}$ & $\begin{array}{l}\text { Vegetation Height }(\mathrm{m}) \\
(2 \text { cases })\end{array}$ & $\begin{array}{l}\text { Return Period (years) } \\
(3 \text { cases })\end{array}$ \\
\hline - Low & - Emergent & RP 100 \\
- Medium & RP 25 \\
- High & & RP 5 \\
\hline
\end{tabular}

Wave attenuation rates from different cases were compared with each other to give an understanding of the relative importance of different hydraulic and vegetation parameters to the wave attenuation process. Some of the results obtained from the variation of vegetation parameters such as vegetation factor and height, for given hydraulic parameters are described below:

1. The model shows an expected overall increase in sharpness of wave attenuation from low density to high density as seen in Figure 4. Due to the nature of wave attenuation processes in the mangroves the curve of the wave attenuation rate becomes flatter anywhere between $300 \mathrm{~m}$ and $800 \mathrm{~m}$ forest width depending on the vegetation factor.

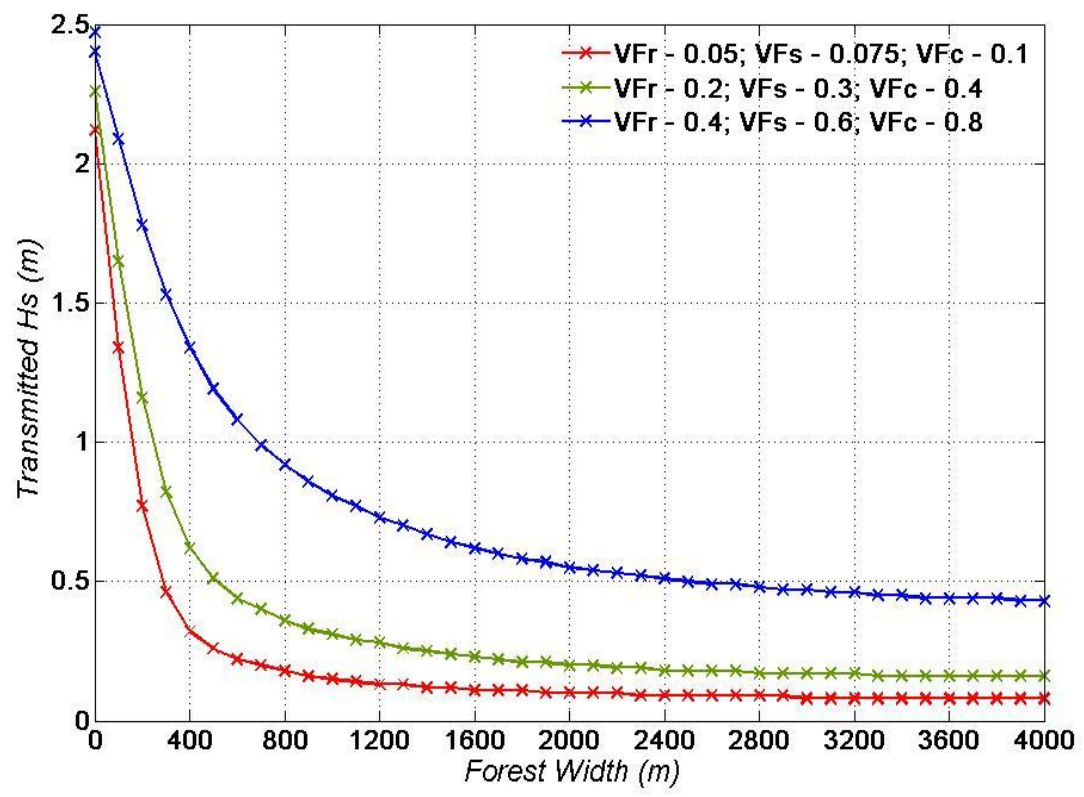

Figure 4: Transmitted wave heights across forest width for varying vegetation factor values for roots (VFr), stems (VFs) and canopies (VFc) with constant hydraulic parameters $(\mathrm{h}=9.6 \mathrm{~m}$. $\mathrm{Hs}=2.5 \mathrm{~m}, \mathrm{Tp}=15 \mathrm{~s})$

2. The generic analysis shows that longer waves initially experience slightly sharper attenuation rates than shorter waves though the rates become almost the same beyond a forest width of around 1000 m, shown in Figure 5. 


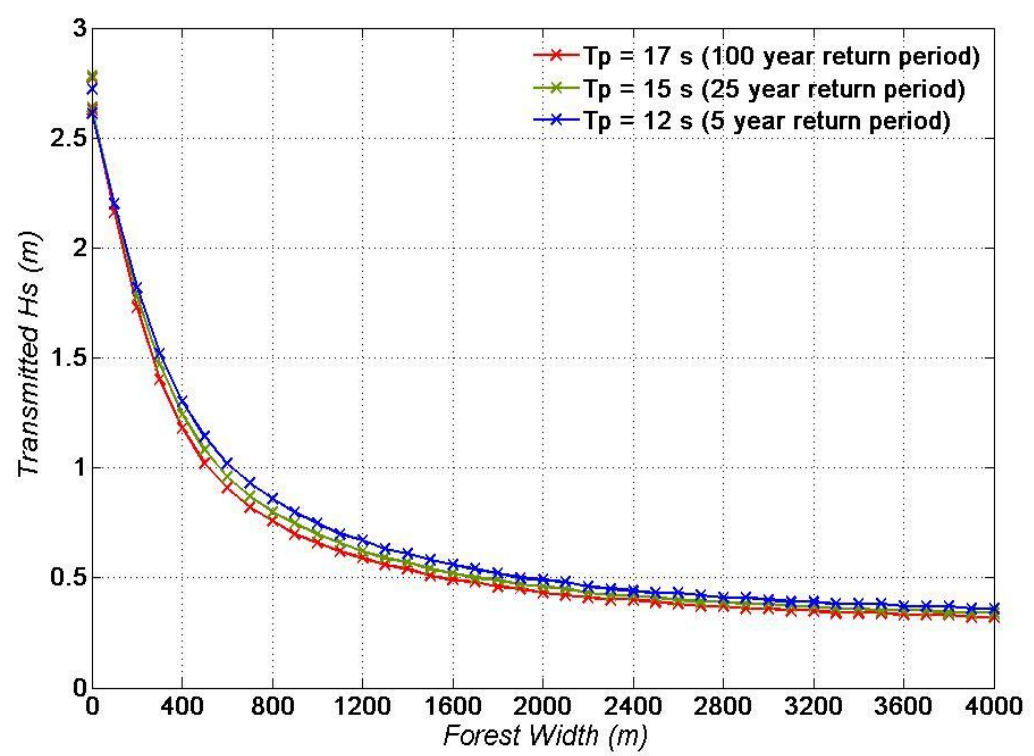

Figure 5: Transmitted wave heights across forest width for varying input time periods with constant wave heights and water levels at medium vegetation factor values

3. The difference between the effects of higher and lower input waves at any specific point was observed to reduce within the vegetation with an increase in the overall vegetation factor, becoming nearly constant for all input wave heights at high vegetation factors as shown in Figure 6.

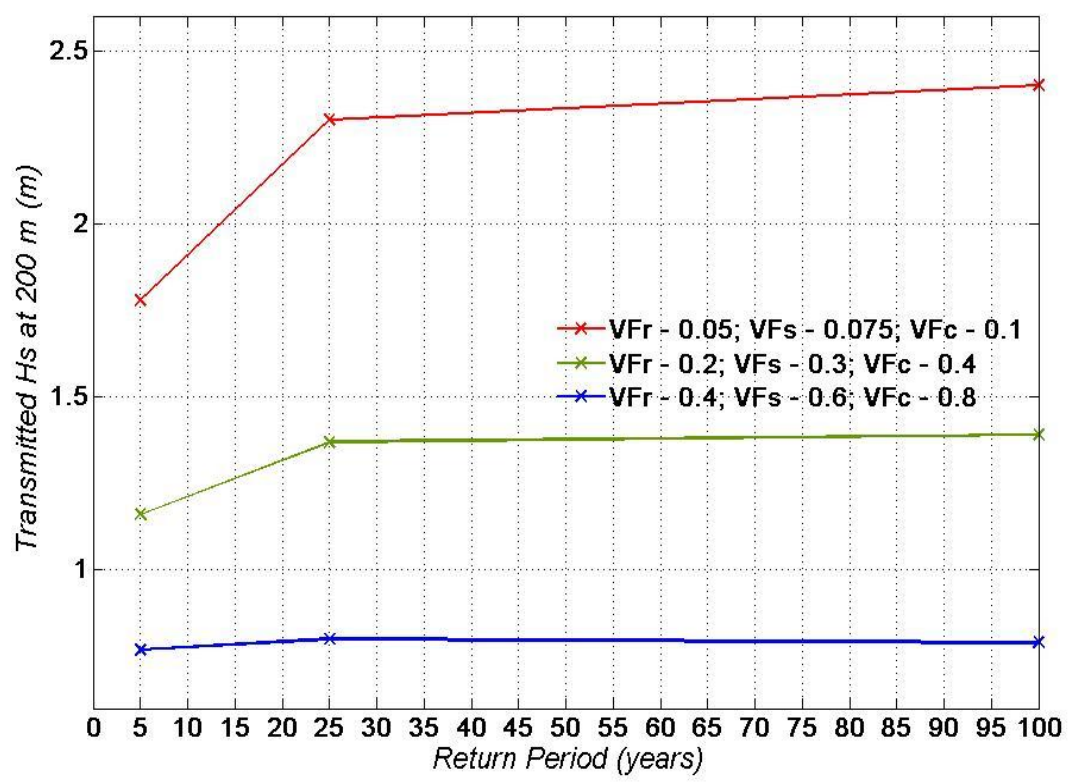

Figure 6: Transmitted wave heights at $200 \mathrm{~m}$ forest width for different vegetation factor values and different input wave heights for increasing return periods (constant $h=9.6 \mathrm{~m}$ and $\mathrm{Tp}=15 \mathrm{~s}$ )

\section{CASE STUDY - KANIKA SANDS}

For the case-study the eastern (seaward) boundary of the 2-D grid for the case study was set at the $11 \mathrm{~m}$ depth contour. The 2-D bathymetry, shown in Figure 7 below was simulated based on a digitized linear bathymetry profile obtained from 1 in 50000 scale navigation maps. 


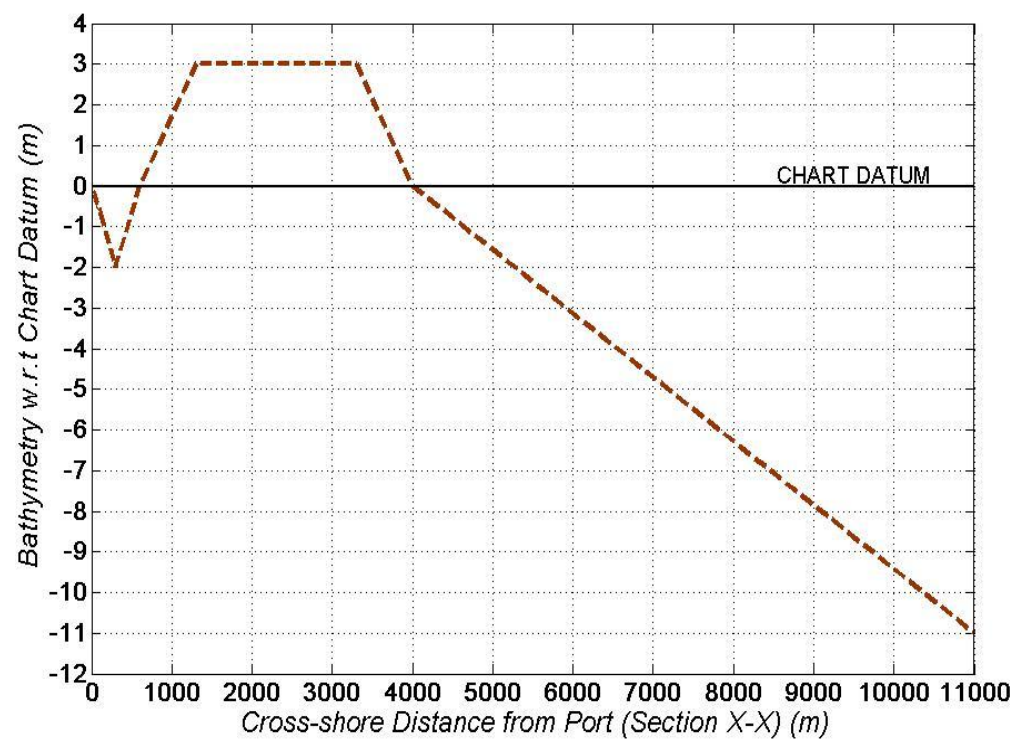

Figure 7: Interpolated near-shore bathymetry for 2-D models with Chart Datum indicated

Grid resolutions for input, computational and output grids were kept constant at $100 \mathrm{~m}$ similar to the grids used in the generic analyses. The windward and seaward sides of the island were assumed to have a slope of around 1 in 200 partly based on the adjacent bathymetry. Sedimentation by mangroves would generally give much flatter slopes in case of an old island. Since Kanika Sands is a relatively new island and is influenced by other large-scale morphological drivers in the region this assumption was thought to be acceptable. The northern and southern boundaries were assumed to be straight and were kept open. The roughly oval mangrove patch with its two extended arms on the northern and southern ends was represented in the form of a rhombus. Vegetation was assumed to exist only on the $3 \mathrm{~m}$ contour.

From the generic analyses it was concluded that the model allows a limited representation of the spatial variation of mangrove species in terms of multiple values of the vegetation density. The spatial vegetation density file was therefore given a uniform value of 1 throughout except in case of the horizontal variation studies). The effect of the difference in wave attenuation rates for different input time periods was considered small enough to be neglected in the case study.

The wave attenuation curve due to medium density vegetation was seen to lie in between the low and high density vegetation curves, as expected. Since the behaviour across vegetation factors follows an expected trend it was decided for the purposes of the case study to model just two scenarios corresponding to 'high' and 'low' vegetation factors. Additionally a 'zero' vegetation factor scenario was included to study the effect of the island in the absence of the vegetation. The values for the 'high' and 'low' scenarios were taken from the assumed realistic values. Three simulations with varying angles of wave attack $-22.5^{\circ}, 45^{\circ}$ and $90^{\circ}$ were run for different vegetation cases for a single hydraulic scenario corresponding to a 25 year event. A scenario with no island and no vegetation and a $90^{\circ}$ angle of attack was simulated to serve as a control case for comparisons. The hydraulic and vegetation parameter values for the different scenarios are listed below in Tables 3 and 4 respectively.

\begin{tabular}{|l|l|l|l|}
\hline \multicolumn{4}{|c|}{ Table 3: Vegetation parameter values for the case study } \\
simulations & \multirow{2}{*}{ Height $(\mathrm{m})$} \\
\hline \multirow{2}{*}{ Layer } & Vegetation Factor & \\
\cline { 2 - 4 } & Case 'LOW' & Case 'HIGH' \\
\hline Roots & 0.05 & 6.5 & 0.5 \\
\hline Stem & 0.075 & 0.6 & 5 \\
\hline Canopy & 0.1 & 2 & 0.5 \\
\hline
\end{tabular}




\begin{tabular}{|l|l|l|l|}
\hline \multicolumn{4}{|c|}{$\begin{array}{c}\text { Table 4: Hydraulic Parameter values for the case study } \\
\text { simulations (constant angle of wave attack } \mathbf{9 0}^{\mathbf{0}} \text { ) }\end{array}$} \\
\hline $\begin{array}{l}\text { Return Period } \\
\text { (years) }\end{array}$ & $\begin{array}{l}\text { Wave Height } \\
(\mathrm{m})\end{array}$ & $\begin{array}{l}\text { Water Level } \\
(\mathrm{m})\end{array}$ & $\begin{array}{l}\text { Wave } \\
\text { Period }(\mathrm{s})\end{array}$ \\
\hline 100 & 9.25 & 12.6 & 17 \\
\hline 25 & 7.44 & 10.0 & 15 \\
\hline 5 & 5.28 & 7.1 & 12 \\
\hline
\end{tabular}

Wave analysis was done along two sections - one in the cross-shore direction across the centre of the island (Section $\mathrm{X}-\mathrm{X}$ ) and one in the alongshore direction at the shoreline along the port (Section Y$\mathrm{Y})$ as shown in Figure 8.
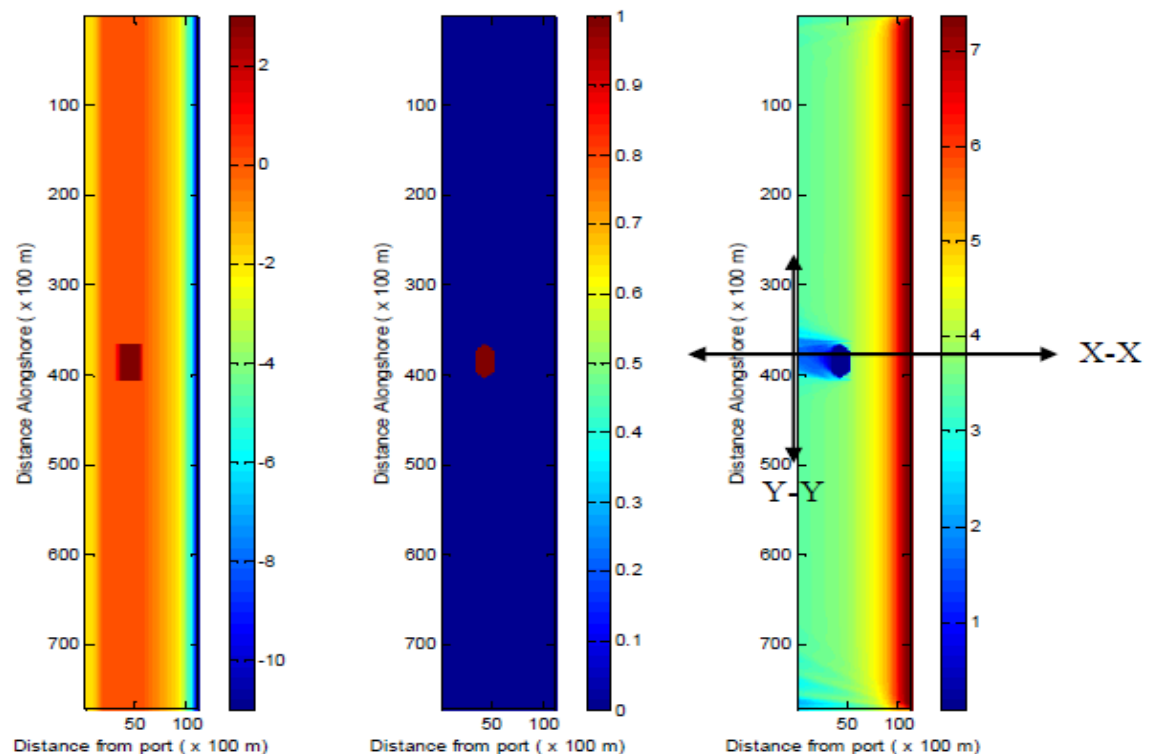

Figure 8: Bathymetry grid (left), vegetation density grid (middle) and transmitted wave heights (right) for an island with high vegetation density and an event of return period 25 years. The two analysis sections cross-shore (Section $X-X$ ) and alongshore (Section $Y-Y$ ) are indicated on the transmitted wave heights grid

1. The analysis of wave heights along Section $X-X$ shows that the presence of vegetation has a considerable effect with regard to wave attenuation in the port for all three return periods. These results are shown in Figure 9 for a return period of 25 years.

2. It is seen from Figure 9 that the vegetation patch is very effective in reducing wave heights at points immediately behind it with a difference of around $0.5 \mathrm{~m}$ being observed between low and high vegetation factor conditions.

3. A relatively sharp recovery of wave heights is seen beyond the vegetation patch with the sharpness increasing for increasing vegetation densities. This phenomenon results in much higher wave heights at the port compared to points immediately behind the vegetation. The diamond shaped island geometry contributes to wave height recovery since it has a smaller shadow region compared to a rectangular island. It can therefore be concluded that the mangrove island has an effect on the port though this is limited by its geometry and its distance from the port.

4. Wave attenuation of nearly $60 \%$ is observed at the port due to the effect of the mangrove island. The attenuation within the island is however nearly $90 \%$.

5. From Figure 10, a $2.5 \mathrm{~m}$ wave height has a return period of more than 60 years under simulated conditions. If the vegetation were removed or destroyed the $2.5 \mathrm{~m}$ wave would occur at least once every 20 years posing the threat of a drastic reduction in the port's design life. The effect of the vegetation and its continued existence are therefore crucial for the design of the port. 


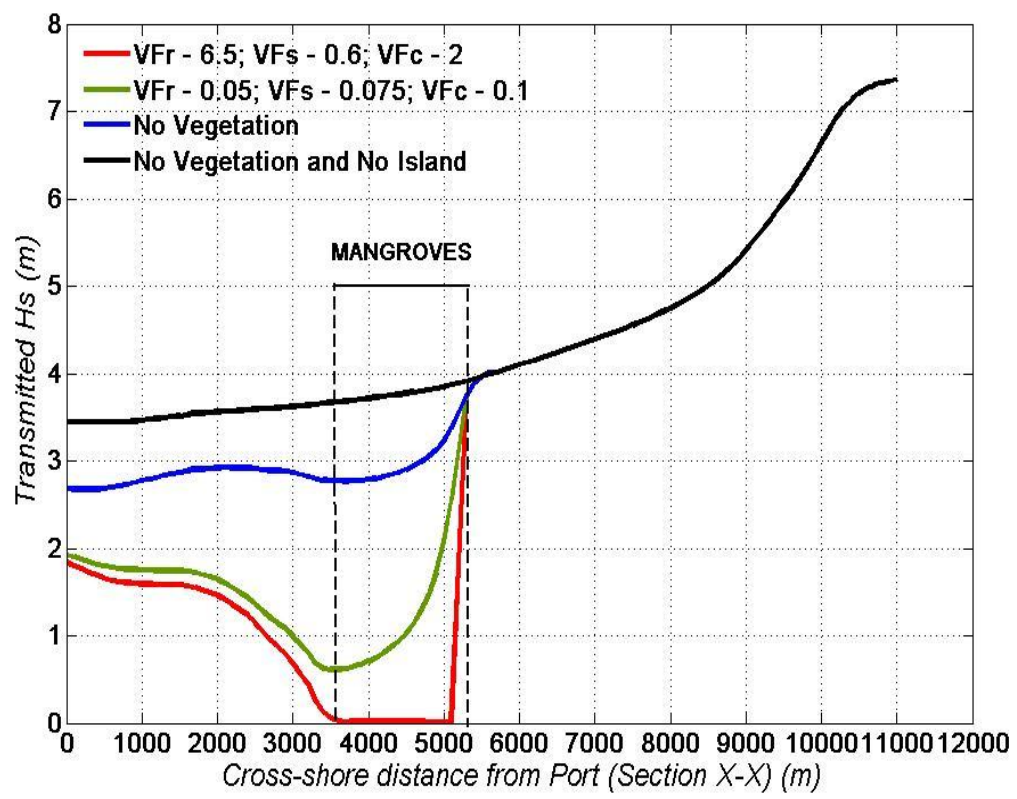

Figure 9: Transmitted wave heights from offshore (right) along Section X-X (cross-shore) for different vegetation factors compared with the 'no veg. and no island' case for a 25 year event

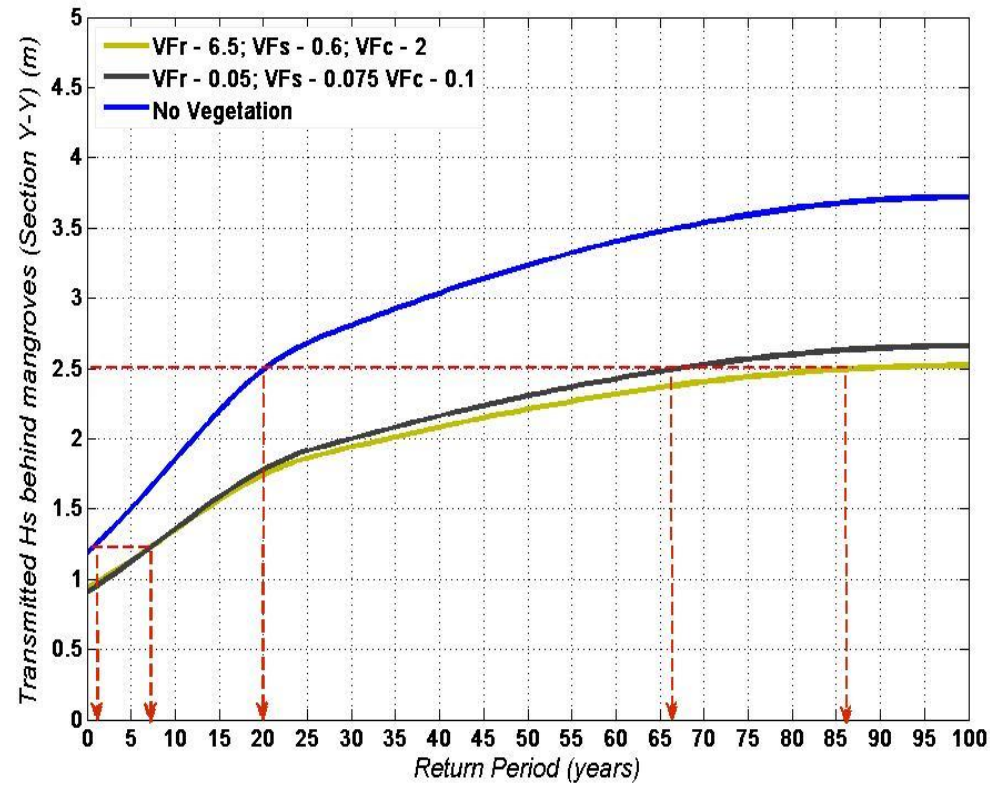

Figure 10: Wave heights at the port at a point directly behind the mangroves versus return periods for different cases of vegetation

Based on the results of the case study and in order to investigate mangrove planting and management options, two studies were conducted that varied the horizontal distribution of vegetationone with an extension of $1 \mathrm{~km}$ each on the northern and southern sides (Case 1) and one with an extension of $2 \mathrm{~km}$ (Case 2) on the northern side and one $\mathrm{km}$ on the southern. In both cases the extension was done for both bathymetry and vegetation for a cross-shore forest width of $500 \mathrm{~m}$. The results from the two simulations for an angle of 22.5 degrees are shown in Figure 11 below. 


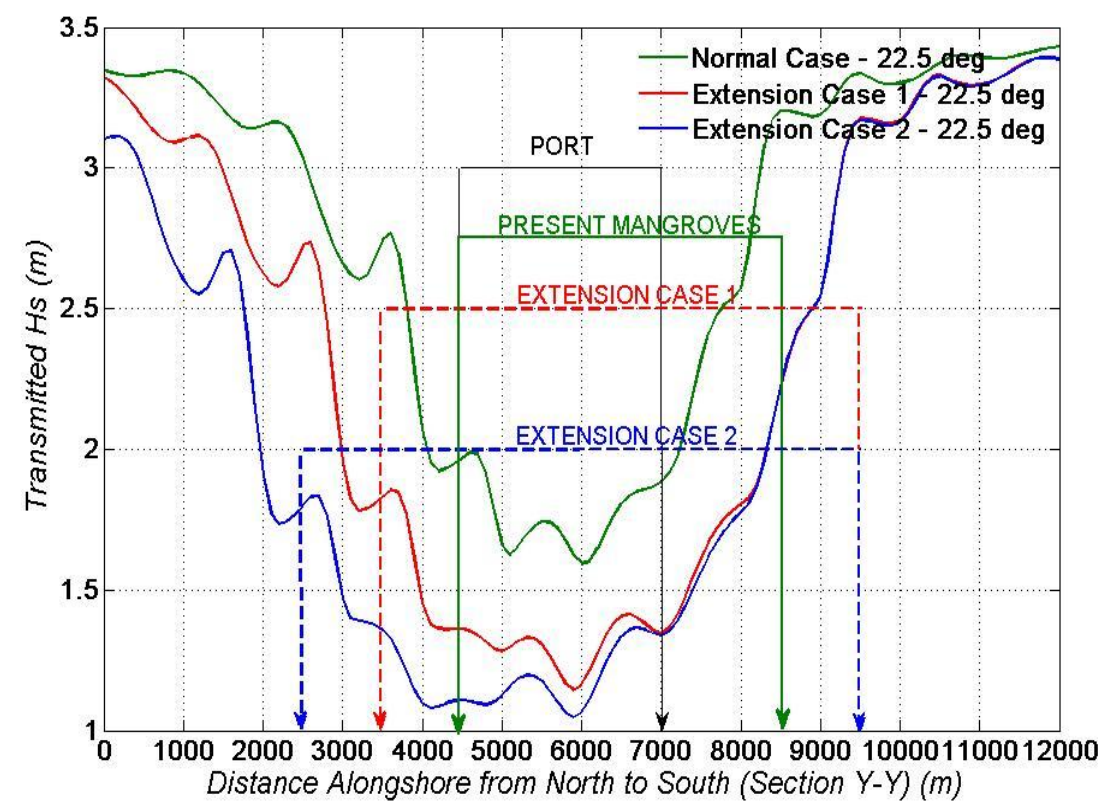

Figure 11: Wave heights at port for original mangroves and the two extension cases at 22.5 deg wave attack angles for a 25 year event. The solid black lines indicate the position of the port, the solid green lines the existing extent of mangroves and the dotted red and blue lines the extent of mangroves for extension cases 1 and 2 respectively

It is seen from Figure 11 that Case 1 shows significant wave height reduction within the port. Case 2 shows even better attenuation with a reduction in transmitted wave height at the port of nearly a metre from the current case. In both cases however the wave heights still remain in the range of 1 to $2 \mathrm{~m}$. The waviness of the curves is thought to be caused by model effects to do with insufficient representation of diffraction effects. These effects were however ignored for the purposes of this analysis. In conclusion, an extension of the island and vegetation on the northern side is highly beneficial to the port though additional protection measures may be needed. Further it would be worthwhile investigating a $2 \mathrm{~km}$ northern extension since this seems to give considerably better protection than a $1 \mathrm{~km}$ extension.

Given that the wave attenuation becomes negligible beyond a certain distance into the mangroves investigations into the effectiveness of mangrove strips were thought worthwhile. While the Kanika Sands island is currently fully inhabited by mangrove vegetation, future strategies for similar islands could look at a 'ring of protection' with mangrove strips. The typical topography of a mangrove ecosystem has an upward slope and it is reasonable to expect that in case of varied vegetation, the mangroves would occupy the seaward portions with the less halophytic plants occupying the landward portions. Also, from a land-use planner's point of view, an island could be inhabited in the centre with a seaward ring or strip of mangroves protecting the central habitat. Finally unintended density reductions due to several reasons could be vital in such a case. With these considerations three simulations were performed. One was for a $300 \mathrm{~m}$ wide mangrove strip with $1 / 10^{\text {th }}$ the 'high' vegetation factor (Case 1) and the second for a $300 \mathrm{~m}$ wide strip with the normal 'high' vegetation factor (Case 2). In both cases there is no vegetation on the rest of the island. The value of $300 \mathrm{~m}$ was chosen from the case studies as being the minimum forest width at which the wave attenuation rate starts decreasing appreciably. Also, a $300 \mathrm{~m}$ width is seen as being practically possible in terms of mangrove planting efforts. The third scenario assumes a $300 \mathrm{~m}$ strip with the 'high' vegetation factor only on the northern, eastern and southern sides with no mangroves on the western (leeward) side of the island (Case 3 ). The simulations were performed for the most extreme event in this study - the 100 year event with all other parameters kept constant. The wave heights for these scenarios compared to the normal scenario with 'high' vegetation factor mangroves throughout the island are shown in Figure 12 below. 


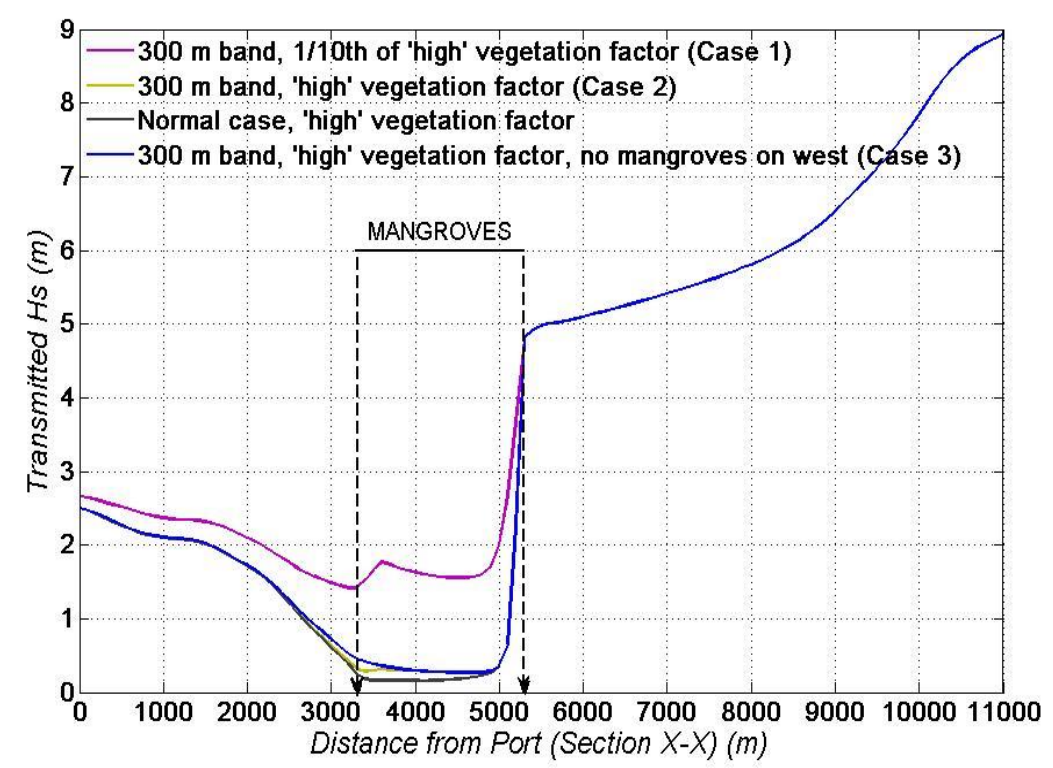

Figure 12: Transmitted wave heights for three vegetation strip cases and the normal case for a 100 year event

From Figure 12 it is seen that for the 'high' vegetation factor, a $300 \mathrm{~m}$ strip has nearly the same effect as the normal scenario with vegetation throughout the island. The planting of vegetation strips therefore seems a far better solution in terms of wave attenuation as it gives the same effect with reduced costs and other difficulties. However, a decrease in vegetation density which is very possible in case of cyclones results in appreciably higher wave heights within the island and up to $800 \mathrm{~m}$ behind it with a smaller difference at the port. This is in contrast with the current situation, where the width of the forest is sufficient to negate the effect of a density reduction. Case 3 indicates that a vegetation strip may not be necessary on the western edge. Here a shadow region is seen up to the edge of the island which may be partly due to numerical effects. Whether this effect is a good approximation of reality needs to be investigated further. Also, the destruction of the western vegetation strip in this case would leave the island and the port completely unprotected. This scenario therefore needs very careful investigation before application.

\section{CONCLUSIONS}

It was concluded from the case study that the mangroves have a definite positive effect on the port in terms of wave attenuation. While the mangrove island's protective effect is limited by its distance from the coast it still causes a nearly $60 \%$ reduction in wave heights at the coast under the given conditions. Though the vegetation may not have a significant effect on daily operations its continued existence is seen as crucial for ensuring the port's safety during its design life. The mangroves have a significant effect only at cross-shore widths greater than 300 to $400 \mathrm{~m}$ and an increase in width beyond $800 \mathrm{~m}$ does not make much of a difference to wave heights at the port. However the current alongshore width of the mangroves might be insufficient in case of waves approaching from the north at angles greater than $45^{\circ}$. The optimum width of the island for maximum protection solely in terms of wave attenuation under the given hydraulic and vegetation conditions therefore ranges from 300 to $800 \mathrm{~m}$ in the cross-shore direction with an along-shore length of around $6 \mathrm{~km}$. These were verified with some horizontal variation simulations. Expansion to the north was thought more relevant given the present conditions. Such an island may be a cheap alternative to a conventional breakwater. Horizontal variation simulations also showed that density reductions on the island due to extreme events do not have much of an effect under present conditions. Further, the planting of $300 \mathrm{~m}$ wide mangrove strips seem a highly feasible and cost-effective substitute to foresting an entire region with mangroves. However, the required thickness of the strip, the densities within the strips and the spacing of the trees would all have to be studied carefully.

Due to the distance of the port from the mangroves and their sizeable width the effect of a change in vegetation density within the simulated range is negligible though there is a marked difference 
between the presence and absence of vegetation. Vegetation density variations have an appreciable effect only beyond a 20 year wave height and only up to a certain distance behind the island. The vegetation heights used in this study ranged from 6 to $10 \mathrm{~m}$ roughly corresponding to a $15-20$ year old forest. From the analyses it may also be concluded that the species $R$. mucronata can offer effective protection against waves at the densities assumed in this study and may therefore be selected where suitable for purposes of artificial mangrove replenishment.

\section{ACKNOWLEDGEMENTS}

This work is based largely on the first author's master's dissertation at the Faculty of Civil Engineering and the Geosciences in TU Delft, The Netherlands as part of the Erasmus Mundus MSc CoMEM course from August 2007 to July 2009. His attendance at the ICCE 2010 was partially funded by Het Lammingafonds in The Netherlands.

\section{REFERENCES}

Burger B., 2005. Wave Attenuation in Mangrove Forests, A Master's thesis publication, Delft University of Technology, Faculty of Civil Engineering and Geosciences, Section of Hydraulic Engineering

Chittibabu P., et al., 2004. Mitigation of Flooding and Cyclone Hazard in Orissa, India, Natural Hazards 31(2), pp 455-485, Kluwer Academic Publishers

Dalrymple R.A. et al., 1984. Wave Diffraction due to Areas of Energy Dissipation. Journal of Waterway. Port, Coastal and Ocean Engineering, 110 (1)

Das S., 2007. Mangroves - A Natural Defense against Cyclones : An Investigation from Orissa, India, South Asian Network for Development and Environmental Economics (SANDEE) Policy Brief 2407

Dube S.K., Chittibabu P., Rao A.D. \& Sinha P.C., 2000. Extreme Sea Levels Associated with Severe Tropical Cyclones Hitting Orissa Coast of India, Marine Geodesy 23 pp 75-90

Fritz H.M. \& Blount C., 2007. Thematic Paper: Role of forests and trees in protecting coastal areas against cyclones, Coastal Protection in the aftermath of the Indian Ocean tsunami: What role for forests and trees? part IV of XII part FAO - RAP Report

Johnston P. \& Santillo D., 2007. The Dhamra-Chandbali Port Expansion Project, Orissa, India A Critique of the Environmental Assessment, Greenpeace International

Kobayashi, N., Raichle, A.W. \& Asano, T., 1993. Wave attenuation by vegetation. Journal of Waterway. Port, Coastal and Ocean Engineering 119, 30-48

Mascarenhas A., 2004. Oceanographic validity of buffer zones for the east coast of India: A hydrometeorological perspective, 2004, Current Science Vol. 86, No. 3

Massel S.R., Furukawa K. \& Brinkman R.M., 1999. Surface Wave Propagation in Mangrove Forests. Fluid Dynamics Research 24, 219-249

Mazda Y., Wolanski E. \& Ridd P.E., 2007. The Role of Physical Processes in Mangrove Environments - Manual for the Preservation and utilization of mangrove ecosystems, TERRAPUB, Tokyo, Japan, pp: 15

Mendez, F.M. \& Losada, I.J., 2004. An empirical model to estimate the propagation of random breaking and nonbreaking waves over vegetation fields. Coastal Engineering 51, 103-118.

Murthy T.S., 2007. Storm Surges in the Marginal Seas of the North Indian Ocean, United Nations/International Strategy for Disaster Reduction (online) Available at: http://www.eird.org/encuentro/pdf/eng/doc15270/doc15270-contenido.pdf [Accessed February 10, 2009]

Narayan S., 2009. The Effectiveness of Mangroves in Attenuating Cyclone - induced Waves, A Master's thesis publication, Delft University of Technology, Faculty of Civil Engineering and Geosciences, Section of Hydraulic Engineering

Selvam V., 2003. Environmental Classification of Mangrove Wetlands of India, Current Science 84(6), March 25, 2003 pp 757-765

Suzuki T. and M. Zijlema. 2010. Wave dissipation by vegetation with layer schematization. Book of abstracts NCK Days 2010.

UNEP-WCMC, 2006. In the front line - Shoreline protection and other eco-system services from mangroves and coral reefs. UNEP-WCMC, Cambridge, UK, 33 pp 